\title{
Changes in properties of polyamide netting materials exposed to different environments.
}

\author{
Sandipan Mondal ${ }^{1 *}$, Saly N Thomas ${ }^{2}$, Manoj Kumar B ${ }^{3}$ \\ ${ }^{1}$ National Taiwan Ocean University, Keelung City 20224, Taiwan \\ ${ }^{2}$ Central Institute of Fisheries Technology, Kochi 682029, India \\ ${ }^{3}$ Kerala University of Fisheries and Ocean Studies, Kochi 682506, India
}

\begin{abstract}
Quality of a material and its durability in different environmental condition are two major factors influence the selection of material for fishing gears. A knowledge on the loss of properties is very useful for taking preventive measure. Present study aimed to find the changes in the properties of polyamide monofilament yarns and multifilament twines exposed to three different environments for a period of 90 days. Significant reduction in both breaking strength $(P<0.05)$ and weight $(\mathrm{P}<\mathbf{0 . 0 5})$ was recorded in the test samples exposed to soil, water $\&$ weathering condition. The multifilament twines had more loss in breaking strength and weight than monofilament yarns in all three environments.
\end{abstract}

Keywords: Polyamide, Monofilament, Multifilament, Breaking strength, Weight loss.

Accepted on June 12, 2019

\section{Introduction}

Polyamide is one of the most important synthetic fibre used for the fabrication of fishing gear. The efficiency of a fishing gear depends, to a very great extent, on the materials used for its construction. In the past natural materials were mainly used in fishing gears. Polyamide (PA) became the first synthetic material to replace cotton/hemp in India for the construction of fishing gear and its indigenous production was started in 1962 [1]. PA is available as multifilament twisted and monofilament single yarn for netting purpose. Previous studies showed the necessitates of frequent replacement of webbing of polyamide monofilament of diameter as thin as $0.16 \mathrm{~mm}$ as this lasts only for 6-9 months [2].

Abdullahi and Saidu [3] reported $18.1 \%$ and $25.7 \%$ of weight loss of nylon 6,6 after a period of nine and fifteen months respectively when kept in soil. Moisture is a very important factor, affecting the mechanical strength of polymers. Polyamides are highly hygroscopic materials because of amine or peptide groups [4]. PA monofilament yarn of very thin diameter is extremely used for fabrication of fishing gears [5]. These nets are often used for one season only and are discarded leading to environmental pollution. To assess the change in properties of netting materials in different environments, a comparative study was taken.

\section{Materials and Methods}

The test material comprised samples of: (i)PA monofilament yarn of $0.23 \mathrm{~mm}$ diameter and (ii)PA multifilament twine of $210 \mathrm{~d} \times 1 \times 2$. Samples were kept in three environmental conditions i.e., water, atmosphere and soil.

\section{Soil}

Polyamide samples were buried in soil at a depth of $20 \mathrm{~cm}$. Samples were tied at $2 \mathrm{~cm}$ interval by vinyl-coated polypropylene (PP) rope fixed on a frame. Samples were buried in the experimental site outside the Department of Fishery Engineering \& Technology, Kerala University of Fisheries and Ocean Studies, Kerala, India. (Latitude of $9^{\circ} 54^{\prime} 44.92^{\prime \prime} \mathrm{N}$ and Longitude of 76¹9'2.67"E).

\section{Marine condition}

An in-situ experiment was conducted by keeping the samples in a glass aquarium of size $180 \times 80 \times 80 \mathrm{~cm}$; having a total volume of $1000 \mathrm{~L}$. The aquarium was filled with seawater collected from Chellanam, Kochi (Kerala) in plastic drums. Samples were sewn into a PP rope $(2.8 \mathrm{~cm}$ diameter $)$. The rope was with non-biodegradable vinyl-coated polypropylene having a diameter of about $280 \mathrm{~mm}$. The rope along with the nylon samples were suspended in the aquarium, the distance between the samples was approximately $5 \mathrm{~cm}$. To avoid large temperature fluctuations the tank was kept inside the laboratory.

\section{Atmospheric condition}

The samples were exposed to atmospheric conditions on the roof top of the Department of Fisheries Engineering Kerala University of Fisheries and Ocean Studies, Kerala for a period of 3 months from February to May 2018. The two ends of the samples were tied to Polypropelene ropes with a $2 \mathrm{~cm}$ gap in between and taking care that there is no tension in the material. The ropes were mounted on a frame of $1 \mathrm{~m} \times 0.7 \mathrm{~m}$. This frame was kept at an area on the roof with sufficient ventilation in order to avoid the build up of excessive heat. This was kept at an angle of $45^{\circ}$ as during the study period there was continuous sunshine on the test site.

Sub-samples from the test material were removed for measuring the change in breaking strength and weight loss after $8,15,30,45,60,90$ days of exposure. The test exposure was done from February, 2018 to May, 2018 when there was almost 
continuous sunshine at the test site. During this period, the mean monthly temperature in the area ranged from 31.6 to $34.1^{\circ} \mathrm{C}$.

The retrieved samples and the unexposed control samples were tested for breaking strength using a Universal Testing Machine (UTM) of model "AG-I $10 \mathrm{KN}$ " as per (1992). Ten replicates of each sample were tested at each sampling and the mean value was taken. Breaking strength after a given period of exposure was calculated as a percentage of the mean initial strength of the corresponding unexposed control sample. The twine is considered unserviceable when the strength is reduced to $50 \%$ of its original value (Brandt, 1959). Weight of each replicate was measured using an electronic balance of model "BP 211 D" before and after the exposure and loss in weight was calculated.

\section{Results and Discussion}

\section{Assessment of mechanical strength property}

The initial breaking strength of the monofilament yarn and multifilament twine were $24.76 \mathrm{~N}$ and $26.7728 \mathrm{~N}$ respectively with the onset of degradation triggered in three environments. The percentage residual tensile strength varied in samples with the monofilament yarns having the highest residual tensile strength in all the three environments than multifilament twines. Results showed reduced breaking strength of samples exposed to all the three environments (Tables 1 and 2). An Analysis of variance (ANOVA) showed consistent reduction in the tensile strength. At the end of the 90 days of exposure, there was significant reduction in breaking strength $(\mathrm{P}<0.05)$ for both monofilament yarn and multifilament twine in all the three environments. Retained tensile strength of monofilament yarns exposed in soil, water and atmosphere were 88.41, 81.41 and $74.23 \%$ respectively (Table 1; Figure 1) at the end of 90 days, whereas retained tensile strength of multifilament twines exposed in soil, water and atmosphere were 84.66, 72.38 and 61.52 respectively (Table 2; Figure 2).

With increase in exposure time the breaking strength

Table 1. Breaking strength of monofilament yarn as a percentage of initial values.

\begin{tabular}{|c|c|c|c|}
\hline Day & In soil (\%) & In water (\%) & In atmosphere (\%) \\
\hline 0 & 100 & 100 & 100 \\
\hline 8 & 97.18 & 92.83 & 89.46 \\
\hline 15 & 95.56 & 91.78 & 87.23 \\
\hline 30 & 93.21 & 89.83 & 84.35 \\
\hline 45 & 92.36 & 87.59 & 80.42 \\
\hline 60 & 89.53 & 84.25 & 77.41 \\
\hline 90 & 88.41 & 81.41 & 74.23 \\
\hline
\end{tabular}

Table 2. Breaking strength of multifilament twine as a percentage of initial values.

\begin{tabular}{|c|c|c|c|}
\hline Day & In soil (\%) & In water (\%) & In atmosphere (\%) \\
\hline 0 & 100 & 100 & 100 \\
\hline 8 & 98.31 & 96.17 & 90.85 \\
\hline 15 & 96.39 & 87.91 & 82.68 \\
\hline 30 & 93.88 & 82.39 & 74.52 \\
\hline 45 & 93.52 & 80.02 & 71.01 \\
\hline 60 & 92.45 & 78.19 & 65.09 \\
\hline 90 & 84.66 & 72.38 & 61.52 \\
\hline
\end{tabular}

J Fish Res 2019 Volume 3 Issue 2
Figure 1. Comparison in the breaking strength of monofilament yarn as a percentage of initial values.

\section{COMPARISON OF CHANGES IN TENSILE STRENGTH}

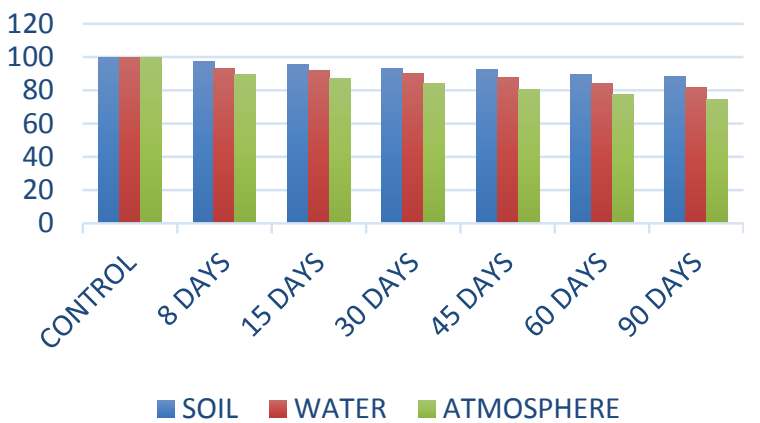

Figure 2. Comparison in the breaking strength of multifilament twine as a percentage of initial values.

\section{COMPARISON OF CHANGES IN TENSILE STRENGTH}

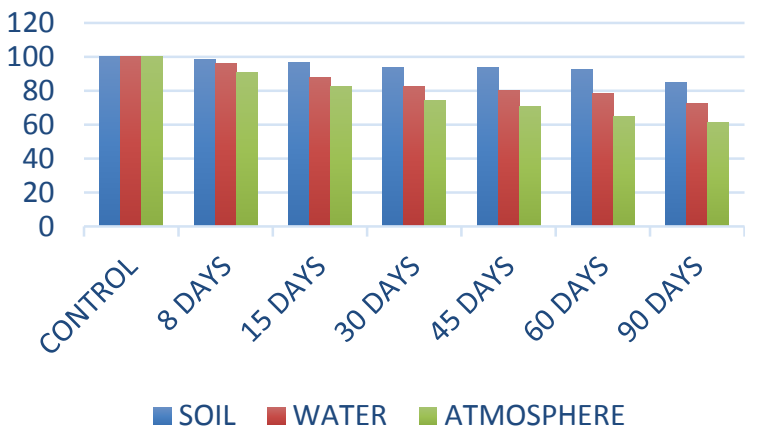

decreased. This was observed in the case of both monofilament yarn and multifilament twine for all three environments. Relationship between breaking strength and exposure time showed a linear relationship when the regression equation fitted to the graph $(\mathrm{R} 2<1$ for both the samples in the all three environments). This shows that the relationship between the rate of deterioration of strength and the period of exposure was linear and this can help in predicting the service life of the material. The present observations were in conformity with those of Meenakumari and Radhalakshmi [6] for the Polyamide multifilament twines induced to photodegradation.

Tensile strength for polyamide multifilament twines of 210 $\times 1 \times 2$ were less than polyamide monofilament yarns of 0.23 $\mathrm{mm}$ diameter in all the three environments (Table 2; Figure 2). This shows that PA multifilament twines were more susceptible to degradation than monofilament yarns. Alsayes et al. [7] and Thomas and Hridayanathan [2] also observed higher degradation by weathering on multifilament material than on monofilament.

\section{Assessment of physical property}

Result on weight loss of samples of polyamide monofilament yarn and multifilament twine exposed to soil, water and atmosphere are given in Tables 3 and 4. Weight loss was more in multifilament twines than in monofilament yarns in all the three environments. An Analysis of variance (ANOVA) showed consistent increase in the percentage weight loss with increase in 
Table 3. Percentage weight loss of monofilament yarn.

\begin{tabular}{|c|c|c|c|}
\hline Day & \% Loss in soil & \% Loss in water & \% Loss in atmosphere \\
\hline 8 & 0.191 & 0.257 & 0.335 \\
\hline 15 & 0.288 & 0.395 & 0.541 \\
\hline 30 & 0.345 & 0.482 & 0.664 \\
\hline 45 & 0.399 & 0.532 & 0.797 \\
\hline 60 & 0.445 & 0.596 & 0.862 \\
\hline 90 & 0.567 & 0.686 & 0.926 \\
\hline
\end{tabular}

Table 4. Percentage weight loss of multifilament yarn.

\begin{tabular}{|c|c|c|c|}
\hline Day & \% Loss in soil & \% Loss in water & \% Loss in atmosphere \\
\hline 8 & 0.221 & 0.287 & 0.376 \\
\hline 15 & 0.317 & 0.361 & 0.509 \\
\hline 30 & 0.373 & 0.402 & 0.776 \\
\hline 45 & 0.405 & 0.485 & 0.915 \\
\hline 60 & 0.436 & 0.541 & 0.985 \\
\hline 90 & 0.667 & 0.797 & 1.13 \\
\hline
\end{tabular}

exposure time. At the end of the 90 days of exposure, there was significant weight loss $(\mathrm{P}<0.05)$ for both monofilament yarn and multifilament twine in all the three environments. Weight loss of monofilament yarns exposed in soil, water and atmosphere were $0.567,0.686$ and $0.926 \%$ respectively (Table 3) (Figure 3 ) at the end of 90 days, whereas the corresponding values for the multifilament twines exposed in soil, water and atmosphere were $0.667,0.797$ and $1.13 \%$ respectively (Table 4) (Figure 4).

The weight lost increase linearly with increase in exposure time indicating that the process is continuous. This was observed in the case of both monofilament yarn and multifilament twine for all three environments. Relationship between percentage weight loss and exposure time showed a linear relationship

Figure 3. Comparison in the percentage weight loss of monofilament yarn.

\section{WEIGHT LOSS COMPARISON}

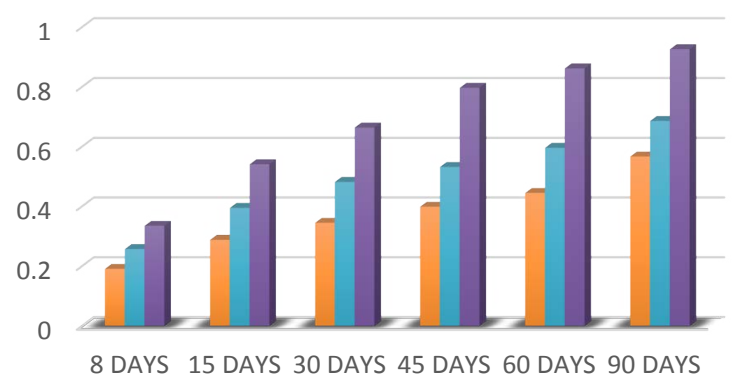

Figure 4. Comparison in the percentage weight loss of multifilament twine.

\section{WEIGHT LOSS COMPARISON}

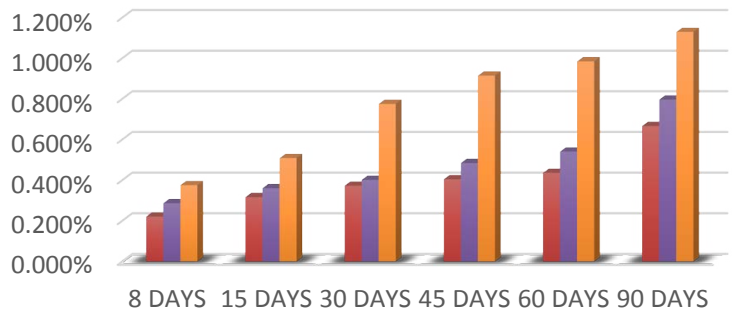

when the regression equation fitted to the graph $(\mathrm{R} 2<1$ for both the samples in the all three environments) which can help in predicting the service life of the material.

\section{Conclusion}

All these results suggest that among all three environments, polyamide fishing net materials are very susceptible to degradation due to exposure in sunlight than other two environments. Among monofilament and multifilament materials, the monofilament is more resistant than multifilament. These studies suggest not to dry the nets in sunlight directly after fishing in view of the strength loss in weathering. Protection of the net from direct sunlight would extent the service life of the polyamide gear material.

\section{Acknowledgement}

The first author wishes to thank Director, Central Institute of Fisheries Technology, Kochi and Vice Chancellor, Kerala University of Fisheries \& Ocean Studies, Kochi for the opportunity to carry out the work.

\section{References}

1. Meenakumari B, Radhalakshmi K, Panicker PA. Netting materials for low energy fishing gear. Fish Technol. Society of Fisheries Technologists. 1993;107-11.

2. Thomas SN and Hridayanathan C. The effect of natural sunlight on the strength of polyamide 6 multifilament and monofilament fishing net materials. Fish Res. 2006;81(23):326-30

3. Abdullahi M and Saidu BT. Biodegradation of plastic using fadama soil amended with organic and inorganic soil. Indian J Sci Res. 2013;4(1):17-24.

4. Pramoda KP, Liu T, Liu Z, He C, et al. Thermal degradation behavior of polyamide 6/clay nanocomposites. Polymer degradation and Stability. 2003;81(1):47-56.

5. Thomas S, John B, Edappazham G, Kalidas C. Standardization of polyamide monofilament yarns for fabrication of gillnets with reference to physical and mechanical properties. J Mar Biol Ass India. 2009;51(1):32-6.

6. Meenakumari B and Radhalakshmi K. Induced photoxidative degradation of nylon 6 fishing net twines. Indian J Text Res. 1988;13:84-6.

7. Alsayes A, Awady El, Awady N. Effects of (UV) irradiation on photochemical degradation of polyamide mono and multifilament twines. Bull Nat Inst Oceanogr Fish. 1996;22:29-41.

\section{*Correspondence to:}

Sandipan Mondal

National Taiwan Ocean University

Keelung City-20224

Taiwan

Tel: 0905020634

E-mail: mondalsandipan31@gmail.com 